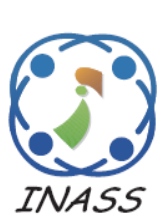

\title{
An Image-based Eri Silkworm Pupa Grading Method Using Shape, Color, and Size
}

\author{
Kiattisin Kanjanawanishkul ${ }^{1 *}$ \\ ${ }^{1}$ Research Unit of Process Design and Automation, Faculty of Engineering, Mahasarakham University, \\ Mahasarakham, 44150, Thailand \\ * Corresponding author’s Email: kiattisin.k@msu.ac.th
}

\begin{abstract}
Eri silkworm pupae are well known as an alternative for a protein food source. At present, they are sold as canned food for long-term preservation. Therefore, good quality and size consistency are essential. To evaluate quality and size, an image-based grading method was proposed. The image of pupae was captured and then shape features (i.e., solidity, aspect ratio, and extent) and color features based on three color models (i.e., RGB, HSV, and L*a*b*) were extracted. Two neural networks with 10 -fold cross validation were separately developed for shape evaluation and color evaluation. After misshapen and discolored pupae were identified by neural networks, remaining pupae were graded into five size numbers according to their length: very small, small, medium, large, very large. Experimental results showed that the average accuracies for shape evaluation and color evaluation were $99.64 \%$ and $99.58 \%$, respectively. The accuracy for size evaluation was $94 \%$. Therefore, the proposed grading method reduces sorting time and increases sorting accuracy.
\end{abstract}

Keywords: Canned pupae, Eri silkworm pupae, Image processing, Neural networks, Pupa grading.

\section{Introduction}

Eri silkworm (Samia ricini) is known as an excellent instance of sustainable agriculture since its cocoon can be used to produce silk and its pupa can be used as a protein food source [1]. Moreover, they have been used for various biomedical applications, e.g., anticancer activity [2], antidiabetic activity [3]. In addition, silk pupa protein hydrolysate is one of several ingredients in cosmetics industries [4].

At present, Eri silkworm pupae are sold as canned food for long-term preservation [5]. Grading is essential since consumers prefer good quality and size uniformity. In general, quality criteria of canned pupae, e.g., moisture content, crispness and color, are affected by two important processing steps in making canned pupae [5], i.e., frying and drying. Frying increases crispness, and reduces moisture content. After frying, pupae are dried in a hot air oven. Since the size of the individual pupa determines the rate of heat transfer for frying and drying, variation in size would cause over-processing or under-processing for frying and drying. Thus, grading is very necessary in order that good quality and size uniformity are guaranteed and grading also simplifies frying and drying processes due to size uniformity.

Traditionally, pupae are graded manually. It is very time-consuming, laborious and inconsistent. A mechanical grading machine has been developed [6] but it cannot detect low-quality pupae which are misshaped or discolored. In addition, the possibilities of pupa damages are rather high. Therefore, the longterm target of our research project is to build an image-based sorting machine that can remove lowquality pupae and sort acceptable-quality pupae based on their size. This machine can be separated into two parts. The first part is an image-based grading module. It takes an image of pupae and uses image processing techniques to grade the pupae based on shape, color, and size. The grading accuracy has been reported in this paper. The second part is the sorting mechanism currently developed.

In the literature, there have been hundreds of research papers using image processing for quality 
Table 1. Pupa size based on length

\begin{tabular}{|c|c|c|}
\hline $\begin{array}{c}\text { Size } \\
\text { Number }\end{array}$ & $\begin{array}{c}\text { Size } \\
\text { Description }\end{array}$ & $\begin{array}{c}\text { Pupa Length: } \boldsymbol{L} \\
(\mathbf{m m})\end{array}$ \\
\hline 1 & very small & $L \leq 20$ \\
\hline 2 & Small & $20<L \leq 24$ \\
\hline 3 & Medium & $24<L \leq 28$ \\
\hline 4 & Large & $28<L \leq 32$ \\
\hline 5 & very large & $L>32$ \\
\hline
\end{tabular}

grading in agricultural and food industry [7-9]. Some of research papers that are related to pupae are given as follows. Zhu et al. [10] used near-infrared (NIR) spectra as signals to discriminate the sex of pupae since they can reflect the difference between male and female pupae at a molecular level. Tao et al. [11] used a convolutional neural network (CNN) model for sex classification of silkworm pupae based on HSI (Hue, Saturation, and Intensity) spectra. Liu and Wang [12] employed a multi-resolution local Gabor binary pattern (MLGBP) feature extraction method and a neural network classifier to identify the gender of silkworm cocoons. Ma et al. [13] used a shortwavelength NIR spectrometer in combination with a partial least squares discriminant analysis to identify female and male silkworm pupae. Raj et al. [14] proposed a multi sensor system for silkworm cocoons gender classification. An image processing procedure was applied to extract shape-related features from each image instance, which, combined with the weight data, were provided as inputs to train a support vector machine-based pattern classifier for gender classification.

Bej et al. [15] proposed an X-ray imaging technique to estimate the silk content and to evaluate cocoon quality without cutting the cocoon. An unsupervised artificial neural network technique was used to classify grades of cocoons into five classes, i.e., good, medium, bad, dead pupa and un-identified quality. Prasobhkumar et al. [16] developed an automated quality assessment system that employed quantitative measurements of size, shape and stain color and automatically classified each cocoon into four defective categories and good cocoons. Liu et al. [17] proposed an algorithm to distinguish stained cocoons from checker cocooning frames. It consisted of an image segmentation and stained cocoon detection based on watershed algorithm and color line chart. Although there have been a large number of research papers related to grading algorithms based on image processing, we believe that our paper is the first work that shows grading results of Eri silkworm pupae using image processing. Shape and color features are extracted from the pupa image and fed into neural networks. Then the good pupae are graded into five sizes, i.e., very small, small, medium, large, and very large. This research problem is very challenging since pupa body is movable and easily damaged.

The paper is organized as follows: section 2 presents general appearance of the pupa and the proposed image processing algorithms based on shape, color and size. Experimental results and discussion are given in section 3 . Then, section 4 is devoted to conclusions.

\section{Materials and methods}

The consumption of Eri silkworm pupae has been reported in many Asian countries, e.g., India, China and Thailand. They are a good source of protein, fat, and minerals [1]. They are also considered a delicacy. The following subsections describe their life cycle and quality based on physical appearance, neural networks, and the proposed image-based grading method.

\subsection{Eri silkworm pupae}

Like other lepidopterans, Eri silkworm has four phases in its life cycle, i.e., egg, larva, pupa and moth. The pupal phase gets started when a larva starts enclosing itself in a cocoon. Inside the cocoon, the silkworm gradually turns reddish brown to dark brown, and the pupal skin becomes harder. At present, there is no international or national quality standard for Eri silkworm pupae. Thus, the local Thai manufacturer of canned pupae (phongphet farm) has established its own quality standard as shown in Table 1. Fig. 1 (a) shows samples of Eri silkworm pupae that are given size number 1 to size number 5 . They are very different in size and mass. Definitely, the same amount of time for frying and drying those pupae is not recommended. Thus, pupae have to be sorted according to their size in order to ease time setting for frying and drying processes.

Besides size, quality is also taken into account. Fig. 1 (a) shows regular pupae, which are reddish, brown and elliptical in shape. Fig. 1 (b) and Fig. 1 (c) show some samples of unacceptable pupae. Evidently, body shape and skin color can be the key features used to distinguish between acceptable and unacceptable pupae. The body shape of a pupa is misshapen or discolored due to improper handling or storing. It can be damaged, if it is cut carelessly during the process of cutting the cocoon in order to take it out. In addition, it turns dark when it is dead or infected during being inside the cocoon. 


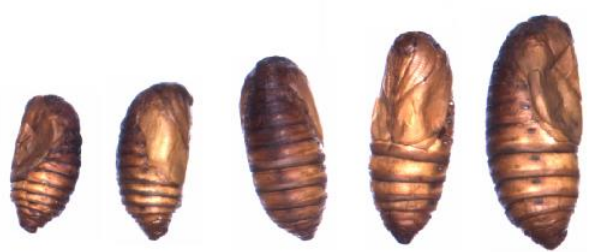

(a)
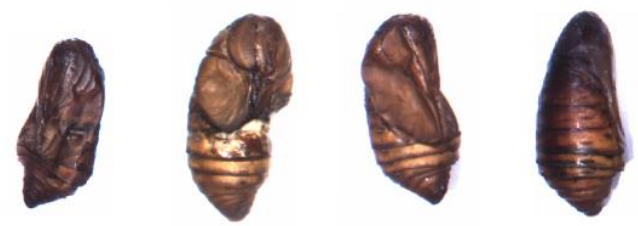

(b)
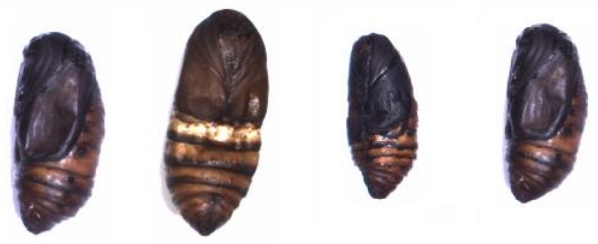

(c)

Figure. 1 :(a) The regular pupae from size no. 1 to size no.5 (from left to right), (b) unacceptable pupae due to misshapenness and (c) unacceptable pupae due to discoloration

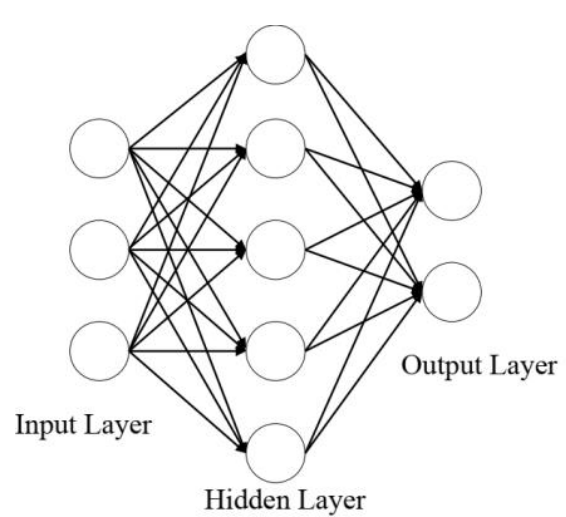

Figure. 2 A neural network model

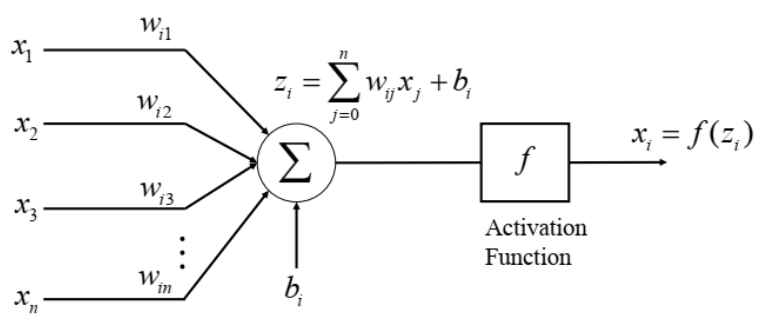

Figure. 3 A model of a single node of a neural network

\subsection{Neural networks}

Neural networks (NNs) have been used to solve a wide range of problems, e.g., object recognition, prediction and optimization. They are represented by a model consisting of a set of nodes along with a set of links between them as seen in Fig. 2. In general, a neural network consists of three layers, an input layer, an output layer and a single hidden layer or multiple hidden layers [18].

Each node has an input/output characteristic and implements a local function. Each input has a weight, see Fig. 3. A larger weight means that the signal is more significant in determining the output. A bias value is added with the weighted sum. Formally, this can be expressed as

$$
z_{i}=\sum_{j=0}^{n} w_{i j} x_{j}+b_{i}
$$

where $w_{i}$ is weight, $x_{j}$ is input, $b_{i}$ is an bias, and $n$ is the number of inputs. $z_{i}$ is the weighted sum to the output neuron and it is fed into an activation function in order to activate the output neuron only if the result is higher than the threshold value.

A learning rule is used to update weights and a bias of the neural network when it simulates in a specific dataset. There are two broad categories: supervised learning, and unsupervised learning. In this work, supervised learning is used. Both the inputs and the outputs are given. First, the network processes the inputs and compares its resulting outputs to the desired outputs. Errors are then propagated back through the system. It causes the system to update the weights. This process is iterative. During the training of a network, the same dataset is processed many times as the connection weights are ever refined. The dataset which enables the training is called the training set.

\subsection{Grading methods}

Since shape and color are used to represent physical quality of pupae and length denotes the size of pupae, an image processing method becomes an excellent choice to assess shape, color, and length on one image. Therefore, the proposed grading method is divided into three steps. First, a neural network is developed to identify misshapen pupae by using shape features extracted from the image. Next, the second neural network is created to identify discolored pupae using average color values derived from various color models. Last, the remaining pupae are graded using their length. The details of each step are given as follows.

\subsubsection{Shape evaluation}

Although there is no formal definition of misshapen pupae, the body shape should be, in general, convex and is elliptical as seen in Fig. 1 (a). As a result, we choose the following features as an input vector of the neural network: solidity, aspect 

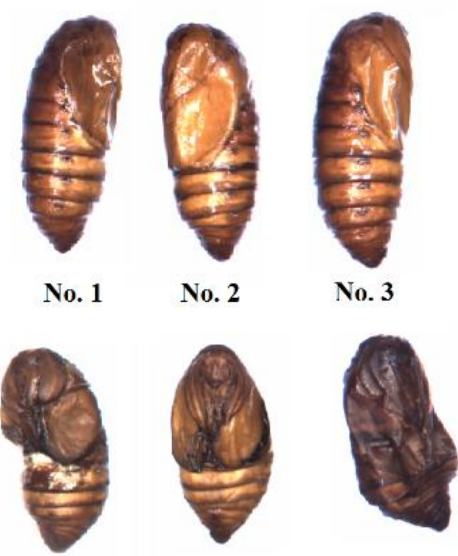

No. 4
No. 5

No. 6
Figure. 4 Pupa samples for shape assessment: three regular pupae and three misshapen pupae

Table 2. Shape features extracted from the image of six regular pupae

\begin{tabular}{|c|c|c|c|}
\hline $\begin{array}{c}\text { Shape } \\
\text { Features }\end{array}$ & No. 1 & No. 2 & No.3 \\
\hline Solidity & 0.966 & 0.972 & 0.969 \\
\hline $\begin{array}{c}\text { Aspect } \\
\text { Ratio }\end{array}$ & 2.529 & 2.475 & 2.632 \\
\hline Extent & 0.915 & 0.906 & 0.927 \\
\hline
\end{tabular}

Table 3. Shape features extracted from the image of six misshapen pupae

\begin{tabular}{|c|c|c|c|}
\hline $\begin{array}{c}\text { Shape } \\
\text { Features }\end{array}$ & No. 4 & No. 5 & No. 6 \\
\hline Solidity & 0.949 & 0.980 & 0.956 \\
\hline $\begin{array}{c}\text { Aspect } \\
\text { Ratio }\end{array}$ & 2.094 & 1.931 & 2.021 \\
\hline Extent & 0.883 & 0.928 & 0.859 \\
\hline
\end{tabular}

ratio and extent. Solidity of an image pupa is calculated using the following equation:

$$
\text { solidity }=\frac{\text { pupa area }}{\text { convex hull area }}
$$

where pupa area is the area of an image pupa given in pixel and convex hull area is the area of convex hull enclosing an image pupa given in pixel. We used the quickhull algorithm [19] to calculate the convex hull.

Although the pupa body is not perfectly convex hull, the solidity is generally close to one. The second shape feature is aspect ratio which is given by

$$
\text { aspect ratio }=\frac{\text { major axis length }}{\text { minor axis length }}
$$

where major axis length is the length of the major axis of the ellipse that has the same normalized second central moments as the image pupa (in pixels), and minor axis length is the length of the minor axis of the ellipse that has the same normalized second central moments as the image pupa (in pixels).

As we examined more than a hundred pupae, the aspect ratio of regular pupae is more than two. The last shape feature is extent of an image pupa, which is calculated by

$$
\text { extent }=\frac{\text { pupa area }}{\text { bounding box area }}
$$

where pupa area is the area of an image pupa (in pixels) and bounding box area is the area of the bounding box surrounding an image pupa (in pixels).

However, in this work, the extent is slightly different to the formal definition. The minimum bounding box (MBB) [20] is first created around an image pupa. Next, the MBB is divided into four sections: one head section, two middle body sections, and one tail section. Then, we calculate the extent for only two middle body sections. The smaller extent is chosen for the shape feature. Obviously, the extent of regular pupae is close to one since the two middle sections of the regular pupa body occupy almost the whole area of the middle two sub-boxes of the MBB. Note that these three shape features do not depend on rotation, translation, and scaling. In addition, they do not rely on the upper half or the lower half of the pupa body.

The detailed algorithm to extract the shape features mentioned above is as follows:

\section{Algorithm 1:}

1. Convert the image to binary using the Otsu's method [21] in order to separate the pupa from the background, and invert white pixels to black pixels and vice versa.

2. Perform the morphological operations: closing, hole filling, and removing of small connected components.

3. Find solidity and aspect ratio.

4. Calculate the minimum bounding box (MBB) around the pupa.

5. Divide the MBB into four sections representing one head section, two middle body sections and one tail section.

6. Compute extent of the two middle body sections and then the smaller one is selected.

As an example, Fig. 4 shows six pupae: three of them have regular shape and the other three pupae are misshapen. After the image of those pupae is processed by the algorithm mentioned above, three shape features are extracted and shown in Tables 2 and 3 . As seen in Table 2, solidity and extent are close 
Table 4. Color features calculated from the color planes of a regular pupa and a discolored pupa

\begin{tabular}{|c|c|c|c|}
\hline \multicolumn{2}{|c|}{ Color Models } & $\begin{array}{c}\text { Regular } \\
\text { Pupa }\end{array}$ & $\begin{array}{c}\text { Discolored } \\
\text { Pupa }\end{array}$ \\
\hline \multirow{3}{*}{ RGB } & Red (R) & 0.507 & 0.273 \\
\cline { 2 - 4 } & Green (G) & 0.337 & 0.216 \\
\cline { 2 - 4 } & Blue (B) & 0.293 & 0.252 \\
\hline \multirow{4}{*}{ HSV } & Hue (H) & 0.197 & 0.743 \\
\cline { 2 - 4 } & Saturation (S) & 0.442 & 0.280 \\
\cline { 2 - 4 } & Value (V) & 0.515 & 0.293 \\
\hline \multirow{3}{*}{ L*a*b** } & $\mathrm{L}^{*}$ & 40.700 & 24.543 \\
\cline { 2 - 4 } & $\mathrm{a}^{*}$ & 16.967 & 8.812 \\
\cline { 2 - 4 } & $\mathrm{B}^{*}$ & 13.858 & -3.053 \\
\hline
\end{tabular}

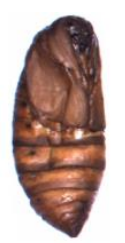

(a)
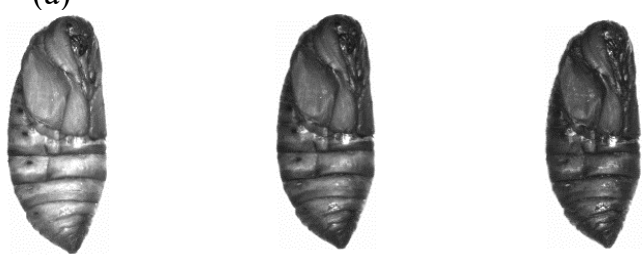

(b)

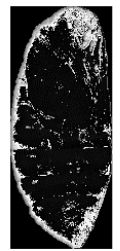

(e)

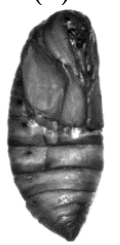

(h) (c)

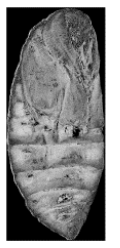

(f)

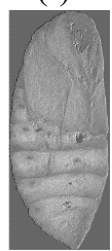

(i) (d)

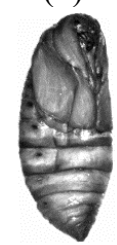

(g)

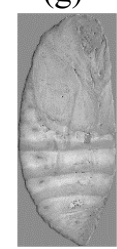

(j)

Figure. 5 An example of a regular pupa: (a) RGB image, (b) red-plane, (c) green-plane, (d) blue-plane, (e) hue-plane, (f) saturation plane, $(\mathrm{g})$ value plane, $(\mathrm{h}) \mathrm{L}^{*}$ plane, (i) a* plane and (j) b* plane.

to one in case of regular pupae and aspect ratio is more than two. In case of no. 5, although the solidity and the extent were close to one, the aspect ratio was very low. The neural network can classify it correctly.

After those three features are extracted, a neural network is used to distinguish between regular pupae and misshapen pupae. The neural network consists of three layers with a 3-neuron input layer and a 2-

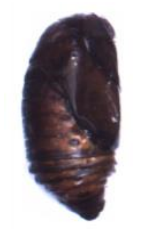

(a)

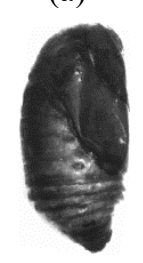

(b)

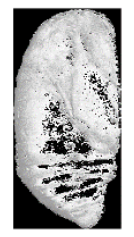

(e)

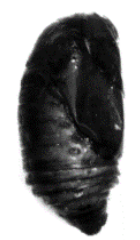

(h)

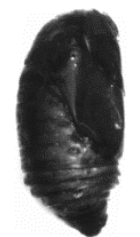

(c)

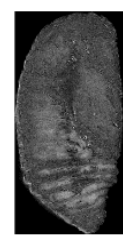

(f)

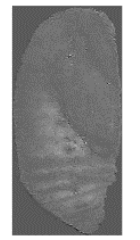

(i)

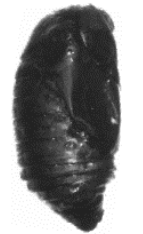

(d)

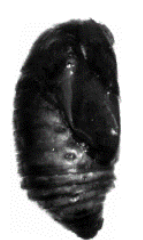

(g)

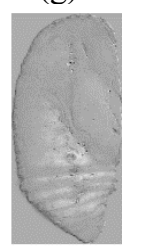

(j)
Figure. 6 An example of a discolored pupa: (a) RGB image, (b) red-plane, (c) green-plane, (d) blue-plane,

(e) hue-plane, (f) saturation plane, (g) value plane,

(h) L* plane, (i) a* plane and (j) b* plane

neuron output layer (two classes). The 10-fold cross validation is applied to obtain the number of nodes in the hidden layer in such a way that the average accuracy is the highest, as seen in the experimental results. A backpropagation learning algorithm used to train the neural network to perform such a task is based on a scaled conjugate gradient algorithm [22]. After this stage, only pupae with regular shape are carried out to the next stage, i.e., color evaluation.

\subsubsection{Color evaluation}

In general, acceptable pupae are reddish brown, thus color values is the good choice to distinguish between acceptable pupae and unacceptable pupae due to discoloration. However, numerical representation of color values depends on the color model we choose. Color models provide a standard method to specify a particular color and each color model reveals different color information [21]. In this work, three color models, i.e., RGB (red, green, blue), HSV (hue, saturation, value), and $\mathrm{L}^{*} \mathrm{a}^{*} \mathrm{~b}^{*}$ are applied to the image of each pupa. As an instance, each color plane of the regular and discolored pupae is extracted 


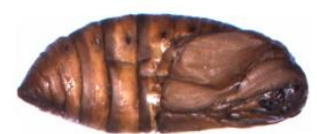

(a)

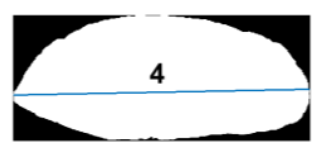

(b)
Figure. 7 Size grading based on an image-based method: (a) a regular pupa and (b) the longest line across the pupa body and the size number of this pupa

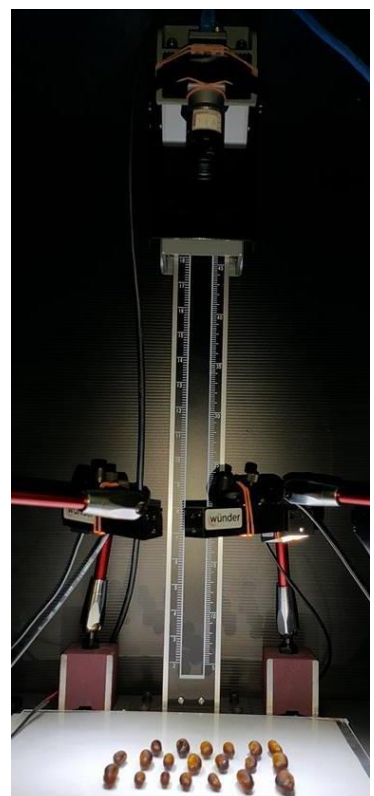

Figure. 8 The experimental setup

and shown in Fig. 5 and Fig. 6, respectively. Then, the average color value of each image pupa is calculated and shown on Table 4. Note that the display of each color plane shown in Fig. 5 and Fig. 6 is linearly scaled to the range from 0 to 255 , for visual purposes only.

The input vector for the input layer of the second neural network consists of nine average color features, i.e., red, green, blue, hue, saturation, value, $\mathrm{L}^{*}$ component, $\mathrm{a}^{*}$ component and $\mathrm{b}^{*}$ component. There are two nodes in the output layer. The number of nodes in the hidden layer is assessed by using the 10fold cross validation in such a way that the highest average accuracy is obtained. A scaled conjugate gradient algorithm is used to train the neural network. Only the regular pupae, which are not discolored, are carried out to the final stage, i.e., size grading.

\subsubsection{Size grading}

Although mass of a pupa should be used for size grading, its length can be adapted due to less time spent on sorting. The following algorithm is conducted to find the length of the longest line across the pupa body and the pupa is then given the size number based on this length (see Table 1).
Algorithm 2:

1. Convert the image to binary in order to separate the pupa from the background, and invert white pixels to black pixels and vice versa.

2. Perform the morphological operations: closing, hole filling, and removing of small connected components.

3. Extract the boundary by removing all interior pixels.

4. Calculate the longest Euclidean distance between two points belonging to the boundary extracted in step 3 and use it to represent the length of the pupa.

5. Convert the length of this longest line in pixel to the actual length of the pupa in mm (note that calibration is performed to find the relationship between the number of pixel and the actual length of the object).

6. Determine the size number based on its length such as the one shown in Fig. 7 (b).

After finishing all three steps above, pupae are sorted into six groups, i.e., very small (size no. 1), small (size no. 2), medium (size no. 4), large (size no. 4), very large (size no. 5), and unacceptable due to misshapenness or discoloration.

\section{Results and discussion}

In the experiment, digital images of pupae were acquired using a GigE CMOS camera (Basler ACE: acA2500-14gm) at resolution of 2592 pixels $\times 1944$ pixels. To avoid interference of environmental light and to reduce effect of shadow, four LED light bars were placed to form a square above pupa samples. Pupa samples were placed on the plate inside the lighting chamber and laid below the camera as shown in Fig. 8. The white background was chosen since discolored pupae were darker.

Since the pupa body exhibits a form of bilateral symmetry, i.e., only one plane divides the body into roughly mirror image halves, we took a photo of pupae in two possible placements. Fig. 9 shows a photo of pupa samples lying on one side, and lying with face down. To show the effectiveness of our proposed algorithms, classification error rate and size grading errors were considered. The classification error rate is the errors that it is a regular pupa but the neural network determines that it is not, or vice versa. The size grading errors are the errors that the algorithm specifies the wrong size number to the pupa. 


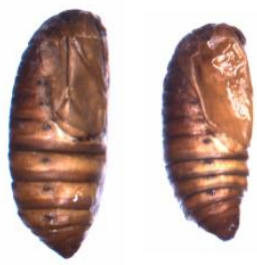

(a)

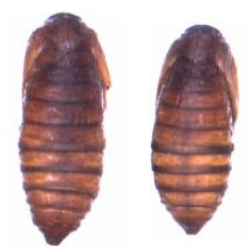

(b)
Figure. 9 Two possible pupa placements: (a) pupae were lying on one side and (b) pupae were lying with face down

Table 5. Experimental results on shape assessment

\begin{tabular}{|c|c|}
\hline $\begin{array}{c}\text { No. of Nodes in the } \\
\text { hidden layer }\end{array}$ & $\begin{array}{c}\text { Average Accuracy } \\
(\mathbf{\%})\end{array}$ \\
\hline 1 & 99.14 \\
\hline 2 & 98.07 \\
\hline 3 & 99.00 \\
\hline 4 & 99.50 \\
\hline 5 & 99.36 \\
\hline 6 & 99.43 \\
\hline 7 & 99.00 \\
\hline 8 & 99.64 \\
\hline 9 & 99.64 \\
\hline 10 & 99.07 \\
\hline
\end{tabular}

Table 6. Experimental results on color assessment

\begin{tabular}{|c|c|c|c|c|}
\hline \multirow{2}{*}{$\begin{array}{c}\text { No. of } \\
\text { nodes in } \\
\text { the } \\
\text { hidden } \\
\text { layer }\end{array}$} & \multicolumn{4}{|c|}{ Average Accuracy (\%) } \\
\cline { 2 - 5 } & RGB & HSV & L*a*b* & $\begin{array}{c}\text { RGB+HSV+ } \\
\text { L*a*b* }\end{array}$ \\
\hline 1 & 98.80 & 99.15 & 98.23 & 99.48 \\
\hline 2 & 98.63 & 99.00 & 98.98 & 99.28 \\
\hline 3 & 98.73 & 99.28 & 99.28 & 99.40 \\
\hline 4 & 98.93 & 99.38 & 99.33 & 99.40 \\
\hline 5 & 99.13 & 99.20 & 98.98 & 99.20 \\
\hline 6 & 98.93 & 99.48 & 99.33 & 99.58 \\
\hline 7 & 98.80 & 99.50 & 99.30 & 99.45 \\
\hline 8 & 99.23 & 99.43 & 99.28 & 99.43 \\
\hline 9 & 98.95 & 99.48 & 99.23 & 99.30 \\
\hline 10 & 99.48 & 98.85 & 98.98 & 99.55 \\
\hline
\end{tabular}

Table 7. Experimental results on size grading: 50 pupae

\begin{tabular}{|c|c|c|}
\hline $\begin{array}{c}\text { Size } \\
\text { Number }\end{array}$ & $\begin{array}{c}\text { Accuracy } \\
\text { (pupae) }\end{array}$ & $\begin{array}{c}\text { Accuracy } \\
(\%)\end{array}$ \\
\hline 1 & 48 & 96 \\
\hline 2 & 47 & 94 \\
\hline 3 & 46 & 92 \\
\hline 4 & 46 & 92 \\
\hline 5 & 48 & 96 \\
\hline \multicolumn{2}{|c|}{ Average } & 94 \\
\hline
\end{tabular}

\subsection{Experiments on shape assessment}

There were 70 regular pupae and 70 misshapen pupae (see Fig. 1 (b)). Three features, i.e., solidity, aspect ratio and extent were extracted from the image of each pupa. To train and to test the neural network, we applied the 10-fold cross validation approach. The whole 140 samples were randomly divided into 10 groups. Eight groups were used for training, one group used for validation and one group used for testing. The process was repeated ten times but with different groups. Therefore, every group was selected for training, validation and testing. Moreover, the number of nodes in the hidden layer was varied from 1 to 10 and then the average accuracy was calculated. As seen in Table 5, the number of nodes in the hidden layer was 8 since the average accuracy was the highest.

\subsection{Experiments on color assessment}

We had 200 discolored pupae (see Fig. 1 (c)) and 200 regular pupae. An image of pupae was taken and each pupa was extracted one by one from the image. We tested four cases. In the first three cases, three color models (i.e., RGB, HSV, and L*a*b*) were assessed separately. Then, all three color models (9 features in total) were combined into one input vector for the neural network. The 10-fold cross validation approach was applied again for all four cases. Table 6 shows the average accuracies of four cases, with respect to the number of nodes in the hidden layer. Apparently, nine features from three color models provided the highest average accuracy with six nodes in the hidden layer.

The main cause of the errors for color assessment was that the back of the upper half were very dark. This resulted in classification errors such that regular pupae were considered discolored pupae. This problem will be further investigated.

\subsection{Experiments on size assessment}

We had 50 pupae for each size number (see Fig. 1 (a)). We used a Vernier calliper to measure the length of each pupa and specified the size number according to Table 1. Then, Algorithm 2 was conducted. Table 7 shows the accuracy of our algorithm. We found that the size grading accuracy was $94 \%$. The main cause of the size grading errors was that some segments of pupa body were movable. Thus, its contraction and expansion made the segment shorter or longer. In addition, its body was not straight. This kind of errors has low impact for size uniformity. 


\section{Conclusions}

Due to delicacy and rich protein content, pupae have drawn much attention over the past few years. At present, they are sold as canned food for long-term preservation. Thus, to maintain size uniformity and quality consistency, an image-based grading method incorporated with neural networks was proposed for grading Eri silkworm pupae into 6 groups, i.e., very small (size no. 1), small (size no. 2), medium (size no. 3), large (size no. 4), very large (size no. 5) and unacceptable pupae. Shape and color features were used as an input vector for the neural networks. The average accuracy for shape assessment and color assessment were $99.64 \%$ and $99.58 \%$, respectively. The accuracy for the size grading was $94 \%$.

Currently, the image-based sorting machine is being developed. The location and the grade of each pupa will be sent to this machine in order to sort pupae according to its grade. It can be used to replace human operators in order to increase consistency, speed, and accuracy in the near future.

\section{Conflicts of interest}

The authors declare no conflict of interest.

\section{Acknowledgments}

This work was financially supported by Mahasarakham University 2016 under Grant No. 5901004. The author would also like to thank Phongphet Farm for supporting pupa samples and providing useful pupa information.

\section{References}

[1] X. Wu, K. He, T. Velickovic, and Z. Liu, "Nutritional, Functional, and Allergenic Properties of Silkworm Pupae", Food Science \& Nutrition, Vol. 9, No. 8, pp. 4655-4665, 2021.

[2] X. Li, H. Xie, Y. Chen, M. Lang, Y. Chen, and L. Shi, "Silkworm Pupa Protein Hydrolysate Induces Mitochondria-Dependent Apoptosis and S Phase Cell Cycle Arrest in Human Gastric Cancer SGC-7901 Cells", International Journal of Molecular Sciences, Vol. 19, No.4, Article ID. 1013, 2018.

[3] S. Chukiatsiri and W. Hangtrakul, "Biological Activities of Protein Extracts from Silkworm Pupae against Noncommunicable Diseases", In: Proc. of International Conf. on Biochemistry and Molecular Biology (BMB2018), Rayong, Thailand, pp. 1-11, 2018.

[4] R. Kunz, R. Brancalhão, L. Ribeiro, and M. Natali, "Silkworm Sericin: Properties and
Biomedical Applications", BioMed Research International, Vol. 2016, Article ID 8175701, 2016.

[5] C. Srikaew and S. Songsermpong. "Eri Silkworm Pupae Processing", In: Proc. of 50th Kasetsart Annual Conf., Bangkok, Thailand, pp. 234-243, 2012.

[6] K. Kanjanawanishkul, P. S. On, P. Thammakit, and J. Chinnakotr, "A rotary Drum Screen with Internal Screw Flights for Eri Silkworm Pupae", In: Proc. of International Conf. on Mechatronics and Mechanical Engineering, Singapore, pp. 14, 2015.

[7] M. Saberioon, A. Gholizadeh, P. Cisar, A. Pautsina, and J. Urban, "Application of Machine Vision Systems in Aquaculture with Emphasis on Fish: State-of-the-art and Key Issues", Review in Aquaculture, Vol. 9, No. 4, pp. 369-387, 2017.

[8] H. Tian, T. Wang, Y. Liu, X. Qiao, and Y, Li, "Computer Vision Technology in Agricultural Automation - A Review", Information Processing in Agriculture, Vol. 7, No. 1, pp. 119, 2020.

[9] M. Meenu, C. Kurade, B. Neelapu, S. Kalra, H. Ramaswamy, and Y. Yua, "A Concise Review on Food Quality Assessment Using Digital Image Processing", Trends in Food Science \& Technology, Vol. 118, Part A, pp. 106-124, 2021.

[10]Z. Zhu, H. Yuan, C. Song, X. Li, D. Fang, Z. Guo, X. Zhu, W. Liu, and G. Yan, "High-speed Sex Identification and Sorting of Living Silkworm Pupae Using Near-infrared Spectroscopy Combined with Chemometrics", Sensors and Actuators B: Chemical, Vol. 268, pp. 299-309, 2018.

[11]D. Tao, G. Qui, and G. Li, “A Novel Model for Sex Discrimination of Silkworm Pupae from Different Species", IEEE Access, Vol. 7, pp. 165328-165335, 2019.

[12]L. Liu and K. Wang, "Automatic Identification System of Silkworm Cocoon Based on Computer Vision Method", Revista CientificaFacultad de Ciencias Veterinarias, Vol. 29, No. 4, pp. 785-795, 2019.

[13] Y. Ma, Y. Xu, H. Yan, and G. Zhang, "On-line Identification of Silkworm Pupae Gender by Short-wavelength Near Infrared Spectroscopy and Pattern Recognition Technology", Journal of Near Infrared Spectroscopy, Vol. 29, No. 4, pp. 207-215, 2021.

[14] A. Raj, R. Sundaram, V. Mahesh, Z. Zhuang, and A. Simeone, "A Multi-sensor System for Silkworm Cocoon Gender Classification via Image Processing and Support Vector Machine", Sensors, Vol. 19, No. 12, Article ID 2656, 2019. 
[15] G. Bej, A. Akuli, A. Pal, T. Dey, and N. Bhattacharyya, "Quality Inspection of Cocoons Using X-ray Imaging Technique", In: Proc. of International Conf. on Control, Instrumentation, Energy and Communication, Calcutta, India, pp. 106-110, 2014.

[16] P. Prasobhkumar, C. Francis, and S. Gorthi, "Automated Quality Assessment of Cocoons Using a Smart Camera Based System", Engineering in Agriculture, Environment and Food, Vol. 11, No. 4, pp. 202-210, 2018.

[17] M. Liu, R. Xu, X. Yan, F. Li, Y. Yan, Z. Song, and H. Song, "Cocoon Image Segmentation and Stained Cocoon Detection Based on Watershed Algorithm and Color Line Chart", International Agricultural Engineering Journal, Vol. 27, No. 3, pp. 356-363, 2018.

[18] C. Mishra and D. Gupta, "Deep Machine Learning and Neural Networks: An Overview", IAES International Journal of Artificial Intelligence, Vol. 6, No. 2, pp. 66-73, 2017.

[19] C. Barber, D. Dobkin, and H. Huhdanpaa, "The Quickhull Algorithm for Convex Hulls", $A C M$ Transactions on Mathematical Software, Vol. 22, No. 4, pp. 469-483, 1996.

[20] G. D. Angelo, and S. Rampone, "Shape-based Defect Classification for Non-destructive Testing", In: Proc of IEEE Metrology for Aerospace (MetroAeroSpace), Benevento, Italy, pp. 406-410, 2015.

[21]R. Gonzalez and R. Woods, Digital image processing, 4th ed., Pearson Education, New York, 2018.

[22] M. Bhatt and T. Patalia, "Neural Network Based Indian Folk Dance Song Classification Using MFCC and LPC", International Journal of Intelligent Engineering and Systems, Vol. 10, No. 3, pp. 173-183, 2017, doi: 10.22266/ijies2017.0630.19. 